\title{
Eye Movement Indices of Post-Saccadic Task Under Cognitive Load: A Path Analysis to Predict Individual's Performance
}

Marzieh Salehi Fadardi

University of Melbourne Newman College

Javad Salehi Fadardi

Ferdowsi University of Mashhad

Monireh Mahjoob ( $\square$ mahjoob_opt@yahoo.com )

Zahedan University of Medical Sciences https://orcid.org/0000-0001-9455-0721

Hassan Doosti

Macquarie University

Research Article

Keywords: Saccades, Eye movement, Task performance

Posted Date: May 18th, 2021

DOI: https://doi.org/10.21203/rs.3.rs-475805/v1

License: (c) (i) This work is licensed under a Creative Commons Attribution 4.0 International License.

Read Full License 


\section{Abstract}

Purpose: The evidence on the linear relationship between cognitive load, saccade, fixation, and task performance was uncertain. We tested pathway models for degraded task performance resulting from changes in saccadic and post-saccadic fixation under cognitive load.

Methods: Participants' $(\mathrm{n}=38$ ) eye movements were recorded using a post-saccadic discrimination task without and with arithmetic operations to impose cognitive load, validated through recording heart rate variability and subjective measurement.

Results: Results showed that cognitive load led to longer latencies of saccade and fixation; more inaccurate responses and fewer secondary saccades $(P<0.001)$. Longer saccade latencies influenced task performance indirectly via increases in fixation latency, thereby, longer reaction times and higher response errors were observed due to limited fixation duration on desired target.

Conclusion: We suggest that latency and duration of fixation indicate efficiency of information processing and can predict the speed and accuracy of task performance under cognitive load.

\section{Introduction}

Individual's performance on visual tasks can be degraded due to task conditions such as cognitive load. Previous investigations in normally sighted individuals have reported that cognitive demands of concurrent tasks can affect saccade eye movements and individuals' performance on visual tasks [1-6]. Fadardi and Abel found both latencies of saccade and fixation on the desired target increased and task performance decreased when participants required to perform a post saccadic task concurrently with an arithmetic task [7]. To see a target, individuals require both to get the eyes on the desired target (fixation) and process the consequent visual information. However, this is not always the case, albeit for normally sighted individuals when reported that they looked at but failed to see a visual target or saw it very late when driving and doing another task at the same time [8]. Accurate responses to visual objects in shortest time may be required under cognitive in real world. We hypothesized that task-induced changes in saccades give rise to individuals' performance degrade on post saccadic tasks indirectly by imposing changes in eye fixation on the desired target. The results of this study have agreed with this notion and suggested that timing aspects of fixation (latency and duration) give rise to, and thus can be used to predict, poor visual task performance.

\section{Methods}

\section{Participants}

Thirty-two graduate students from the University of Melbourne (24 females and 8 males) with age 30.81 \pm 12.5 years participated in the experiment. All participants had normal general health condition and 
visual acuity $\geq 6 / 9$ in each eye. Participants were requested to avoid sedatives and alcohol consumption the night before the tasks.

\section{Apparatus and Procedures}

All procedures contributing to the current study conformed to the Declaration of Helsinki and were approved by the local ethics committee at The University of Melbourne. All participants volunteered for the experiment, and the signed written informed consent was obtained from them.

The tasks were presented using SR Research Experiment Builder 1.6.2 (SR Research, Mississagua, Ontario) and a $1024 \times 728$ NEC-WT610 projector with a screen placed at $160 \mathrm{~cm}$ distance from participants. Participants were required to look at a fixation point presented at central gaze position until it disappeared in a random time between $1500-3000 \mathrm{~ms}$. The fixation point was followed by a Tumbling E target presented randomly across $\pm 25^{\circ}$ horizontally away from center in $5^{\circ}$ steps. Participants were required to look at and promptly discriminate the direction of the $E$ target (right or left) using a game player console. Participants were highly encouraged to do the tasks with the highest accuracy and speed as much as possible. Submission of manual responses followed by the fixation target re-appeared and participants required to look back to the center and wait for another $E$ target to be presented. Size of the Tumbling E and fixation targets were the same; i.e., 0.3 LogMAR. Each eccentric gaze position was tested five times.

The task was repeated with concurrent arithmetic task to impose a high level of mental load within participants. For arithmetic task, the participants were required to continuously subtract 7 from a number between 100 and 200 and verbalized arithmetic responses in $0.5 \mathrm{~s}$. Participants' performance on the arithmetic task recorded and was qualitatively monitored after the experiment terminated. Task performance was measured using participants' reaction time to the $E$ targets and percent rate of accurate responses manually submitted. The time-related variables including participants' reaction time and latency of eye movements were measured using the onset time of each target on the screen; that was determined by installing a photocell on a corner of the screen which recorded luminance changes.

Eye movements were recorded during the tasks using an EyeLink II high-speed head mounted video eye tracker (SR Research, Mississagua, Ontario) at a sampling rate of $500 \mathrm{~Hz}$ in pupil tracking mode with an accuracy of $0.5^{\circ}$ or better. Head movements were minimized during the tasks using an adjustable supportive headrest. Participants' head movements were also qualitatively evaluated offline by plotting the positions of the head markers of the EyeLink II.

Concurrent heart rate recordings and a retrospective subjective rating score were used to confirm withinsubjects' variation in level of cognitive load across the task conditions. The workload score was derived based on ratings on Mental Demand, Temporal Demand, and Effort subscales (MTE score) using a computer version of NASA TLX (Task Load Index) within 15 min after the tasks [9]. Heart rate was recorded using non-invasive plethysmograph transducer ear clip (MLT1020, ADInstruments, Australia). To minimize recording artifacts, a piezo-electric pulse transducer (MLT1010, ADInstruments) attached to 
each participant's left thumb and were used to qualitatively monitor participants' physical movements. Heart rate was calculated using MATLAB R2012a (The Mathworks, Natick, MA) and Kubios HRV2 software.

\section{Eye Movements Analysis}

Using the analogue output from the EyeLink II (resampled at $1000 \mathrm{~Hz}$ ) allowed us to synchronize the time of the eye movements with the target timing. The digital output from EyeLink II was used for the analysis of eye position in MATLAB R2012a after being digitally low-pass filtered with a cutoff at $70 \mathrm{~Hz}$. A bidirectional filter (using the Matlab filtfilt command) was applied to the data to avoid time delays. The filter used a mean squares algorithm to produce the least error signal; i.e., the difference between the desired and the actual signal. Velocity data were derived from the position data and smoothed using a second order differentiator with a cutoff frequency of $62.5 \mathrm{~Hz}$.

Saccades were selected using a custom written MATLAB program that presented potential saccades for manual review and selection. The program used the criteria of $10 \% \mathrm{~s}$ and $8000 \% \mathrm{~s}^{2}$ for saccade onset and offset after target step. Trials associated with blinks, predictive saccades, or saccades with directional error or gain of less than 0.5 or greater than 1.5 were not included in analysis [10]. For each participant, asymptotic peak velocity, saccade latency and gain were measured under each task condition. Occurrence of any secondary saccade was also marked for each trial.

Latency of initial eye fixation, also termed target acquisition time [7], was determined as the time between target onset on the screen and initial placement of the eye gaze within $0.5^{\circ}$ of target of interest where was typically located at the end of the saccadic eye movements. Trials with fixation losses due to saccades larger than one degree were not included in the analysis. Fixation duration was measured as the time duration of fixating on the target before a manual response to the target, right or left, was submitted.

\section{Data Analysis}

Task conditions were compared within participants for any significant effect on task performance, saccade eye movements; i.e., latency, gain and velocity of prosaccades, probability of occurrence of secondary saccades, latency and duration of eye fixation on the desired target. Correlation and regression tests investigated potential relations between within participants' changes in multiple variables including task performance, cognitive load and eye movement variables. At the next step, path analysis (standardized regression coefficients and correlation between variables) were used to find a model for which the changes in load indicators, affected visual task performance through possible changes in saccades and/or fixation eye movements. The Chi square and its corresponding $P$-value of 0.05 were used as the criteria to exclude a variable from the model. Acceptable model was indicated using the criteria of 0.90-1.00 for Comparative Fit Index (CFI) and 0.00-0.06 for Root Mean Square Error of Approximation (RMSEA). The models with largest CFI and smallest RMSEA were determined as the final path models for the effects of cognitive load on participants' reaction time and response errors submitted for the discrimination task. The IBM SPSS with AMOS Graphics Versions 24 (IBM Corporation, Somers, NY) were used to analyze data. 


\section{Results}

Both rating score of mental load and heart rate significantly increased within participants during the task with mental arithmetic when compared to the task without arithmetic (mean difference (SE); for the load score: 15.77 (1.17), $P<0.001$.; for heart rate: 10.25 (1.17) bpm, $P<0.001)$. We hence confirmed that the level of cognitive load significantly increased during the task with arithmetic. Figure 1 shows the changes in heart rate, saccade variables and task performance from the low to high load within participants. Latencies of saccade and fixation increased significantly during the high load when compared to the low load (for saccade latency: 37.37(32.14) ms, $P<0.001$.; for fixation: $40.86(8.85) \mathrm{ms}, P<0.001)$. Saccade gain was just significantly greater for the high load when compared to the low load $(0.029(0.011), P=$ 0.042). Probability of occurrence of secondary saccades was less under the high load than under the low load $(0.123(0.024), P<0.001)$. Inaccurate responses submitted on discrimination task significantly increased under the high load when compared to the low load (10.8\% versus $0.97 \%$; Wilcoxon Signed Ranks Test: $P<0.001)$. Fixation duration tended to decrease with the high load, however, the changes in fixation duration and participants' reaction time to the targets were not significant with cognitive load (fixation duration under low load: 391.33 (40.36) ms; and under high load: 348.29 (11.95) ms.; participants' reaction time under low load: 766.49(10.92) ms; and under high load: 785.04 (35.89)).

An inverse correlation was found between changes in fixation duration and response errors from the low to high load $(\mathrm{r}=-0.54, P=0.004)$. A significant correlation was found between participants' reaction time to the $E$ targets and latency of fixation on the desired target $(r=0.313, P=0.015)$. The more response errors submitted for the discrimination task was significantly associated with less probability of occurrence of secondary saccades $(r=-0.401, P=0.001)$. The correlation between the changes in task performance metrics, i.e., reaction time and accuracy of manual responses, from the low to the high load just failed to be significant $(r=-0.503, P=0.05)$.

Path analyses were employed to investigate potential relationships among the task-induced changes in heart rate, MTE score, task performance and eye movements variables, i.e., latency, gain and velocity of saccades, latency and duration of fixation, occurrence of secondary saccades. Figure 2 illustrates the best path models for the effects of task condition on performance metrics including participants' reaction time (Chi square: $0.098, \mathrm{df}=2, P=0.952$, RMSEA: .000, NFI = .031), and response errors (Chi square: $6.334, \mathrm{df}=10, P=.786$; RMSEA: $.000, \mathrm{NFI}=.737$ ). For both models, changes in the task condition was best indicated by heart rate and showed negative effects on individuals' task performance via changes in saccade latency. Changes in heart rate, used as the metric of cognitive load, showed an increasing effect on saccade latency (standardized regression weight $(\beta)=0.07$ ). There was an inverse relationship between saccade latency and the probability of occurrence of secondary saccades $(\beta=-0.18)$. The increase in saccade latency was found to delay fixation on the desired target $(\beta)=0.18$ ) which showed an increasing effect on participants' reaction time $(\beta)=0.08)$. The delay in fixation was found to limit fixation duration $(\beta)=-0.32$ ). Shorter duration of fixation was found to contribute in probability of submission of inaccurate responses increased on discrimination task $(\beta)=-0.59$ ) 


\section{Discussion}

Results obtained by the current study showed cognitive load can affect individuals' task performance at post saccadic position indirectly via changes in eye movements. Increase in saccade latency with high cognitive load can degrade visual task performance indirectly by increase in fixation latency and decrement in duration of fixation on desired target.

Longer saccade latency as found in the current study agreed with the previous findings for the effect of cognitive demands on saccade latency [5]. Eye movements and attentional processing required in cognitive tasks are integrated tightly at the parietal, frontal, and temporal lobes [11, 12]. Attention and saccade tasks result in activation of the bilateral dorsal parietal lobes, frontal and the supplementary eye fields [12]. Programming for saccades, visual detection or recognition tasks are closely coupled $[13,14]$. Arithmetic tasks activate broad brain areas including front-parietal areas which are also triggered during eye movements $[12,15]$. Stuyven suggested that saccades towards eccentric targets are not generated purely involuntarily and can be executed in a controlled way [5]. Controlled attention to the tasks which involve similar neural resources, as used here, reduces the available capacity for information processing and results in the failure to respond appropriately $[4,10,12,16-20]$.

Post saccadic visual tasks such as discrimination, have been found to increase the probability of secondary saccades [21]. However, probability for secondary saccades decreased under the high load possibly because attention allocated to the discrimination task reduced by the concurrent arithmetic task. The nature of secondary saccades is reflexive [22]. Meanwhile, controlled execution of saccades can suppress reflexive saccades under high load condition $[4,17]$. In addition, programming of secondary saccades is prior to their execution and during latency of initial saccades [23]. Like initial saccades, secondary saccades contribute to target acquisition time and final eye position [24]. Under cognitive condition, cost-effective programing of saccades might encourage saccade system to fixate on desired target more by using a single saccade rather than saccade sequences. Despite changes in occurrence of secondary saccades with the high load, we did not find any effect of secondary saccades on visual task performance.

Longer saccade latency resulted task performance decreased, however mainly by increase in latency of fixation on the desired target. According to Fig. 2, a delay in eye fixation on the target can follow a delay in individual's reaction time to the target; i.e., being slow in reacting to visual stimulus. Our results are consistent with the findings of Wang et al. who examined eye movements in a group of patients with involuntary continuous ocular oscillations among whom complain of being slow to see is common (infantile nystagmus) [25]. Being slow to see can be reported also by normally sighted individuals when, for example, drivers may report that they looked but did not see or saw an object late [8]. Being slow to see has been identified clinically as prolonged visual recognition time; i.e., individuals' reaction time to visual targets as used here. Investigations of eye movements and reaction time in both these patients and normally sighted individuals, agreed that longer time taken to respond to a visual target at post saccadic position, is mainly resulted from increased time taken to fixate on desired target rather than 
longer saccade latency or duration of fixation on the target $[7,26]$. Thus, an increase in recognition time is predicted to be following increase in latency of fixation; that is also shown by our results for changes in timing of eye movements and participant's reaction time from low to high load (Fig. 2).

Despite associations between changes in task performance and latency of eye movements, our results did not show any significant difference in the task conditions for participants' reaction times and fixation durations. Previous investigations have suggested two behavioral profiles for the discrimination tasks with mental arithmetic; those include focusing on the accuracy or speed of responses at the expense of the other $[27,28]$. One possible reason for no significant change in our participants' reaction time can be the perceived task urgency might result in the participants concerned more about speed of their performance rather than accuracy of their responses. Task urgency and accuracy also affect saccade latency albeit via different mechanisms $[2,29,30]$; i.e., perceptual urgency can lower threshold of saccade execution while the former one can affect saccade latency via information processing system [18, 31]. It seems that the amount of the enhancement in saccade execution due to perceived urgency, might not large enough within our participants and thus saccade latency increased under cognitive load of concurrent arithmetic task. Saccades are susceptible to attentional disruption when they need to compete with attention to allocate the required capacity of information processing $[5,16,19,20,32]$.

According to the previous investigations, prolonged visual recognition time seem to be unlikely because of the speed of information processing being slow $[7,26]$. Using a simulated driving task concurrently with phone conversing, Recarte and Nunes measured longer latency of fixation, and more individuals' response errors with no significant change in the participants' reaction time [33]. Consistently, Fadardi and Abel suggested efficiency of information processing, despite no significant change in its duration, gives a rise to resolve target details during fixation [7]. The previous results suggested that performance was not degraded due to the shortage of time but resulted from poor information processing $[7,33]$. According to our results, decrement in fixation duration predicted more response error in individual's task performance (Fig. 2). On the other words, longer fixation duration is expected to provide a correct response under cognitive load. Our results are consistent with previous findings by Tsai et al. who used auditory arithmetic concurrently with a simulated driving task [34]. The results of them showed fixation duration did not change across cognitive levels but could predict upcoming errors when fixation duration decreased just before an error in the arithmetic task [34]. The results obtained by scene viewing studies have shown that information extracted during a fixation affects the onset timing of following saccade to terminate that fixation [35-37]. Cognitive level of a scene viewing task have been found to increase fixation duration [37]. However, the processing difficulty used were relevant to the viewing image. Task design and within subjects' comparisons as used in the current study make the target features unlikely affect our results obtained for fixation duration. Attention and oculomotor processing are integrated tightly in the neural level of the parietal, frontal, and temporal lobes $[11,12,38]$. The efficiency of information processing can be reflected in participants' task performance in terms of both speed and accuracy of the responses $[27,28]$. According to our results, it seems that providing a correct response requires longer time duration in presence of limited capacity of information processing or the quality of processing may be degraded despite the presence of healthy oculomotor system. The visual targets used 
in the current study were above the participants' visual thresholds. The impairment of visual processing with cognitive load might be well greater with a high visually demanding target; an example for this would be reading a faded small printed sign while conversing during a drive behind schedule. However, further studies may address it.

\section{Conclusion}

It seems that to gain efficient outcomes on post saccadic tasks, both latency and duration of fixation seem to be required to be well managed. The current study suggests that latency and duration of fixation on desired target can respectively indicate how slow and well an individual can response to a postsaccadic target. The current study showed that cognitive load changed individuals' response errors and reaction time indirectly through changes in saccade latency. Visual tasks under high cognitive load conditions can limit application of top-down processes and result in poor task performance. Cognitive load can affect reflexive responses of visual system, e.g. secondary saccades decrease, latency of saccades increases and longer time is required to get the eyes on the desired target. Distracted minds are a possibility in daily activities when both accuracy and speed are required to deal with visual targets under a high load situation such as driving. In such situations, increased latency of saccade can give rise to late eye fixation and degraded efficiency of information processing during fixation. Latencies of saccade and fixation can be used to monitor forecasted performance especially when both time and accuracy matter.

\section{Declarations}

\section{Acknowledgments:}

Some data of the current study were used in a previous study by Fadardi \& Abel 2018.

\section{Funding}

This work was supported by The University of Melbourne.

Conflict of Interest: No conflicting relationship exists for any author.

\section{References}

1. Drewes J, VanRullen R (2010) Ongoing EEG oscillations and saccadic latency. J. Vis 10(7): 508-508.

2. Drewes J, VanRullen R (2011) This is the rhythm of your eyes: the phase of ongoing electroencephalogram oscillations modulates saccadic reaction time. J. Neurosci 31(12): 4698-4708.

3. Fadardi M. S, Abel L. A (2012) The Effect Of Cognitive Load On Saccadic Charactristics. Invest Ophthalmol Vis Sci 53(14): 4865-4865.

4. Roberts R. J, Hager L. D, Heron C (1994) Prefrontal cognitive processes: Working memory and inhibition in the antisaccade task. J. Exp. Psychol. Gen 123(4): 374. 
5. Stuyven E, Van der Goten K, Vandierendonck A, Claeys K, Crevits L (2000) The effect of cognitive load on saccadic eye movements. Acta Psychol 104(1): 69-85.

6. Mahjoob M, Anderson AJ (2019) Contrast discrimination under task-induced mental load. Vis. Res 1(165):84-9.

7. Fadardi M. S, Abel L. A (2018) Saccades under Mental Load in Infantile Nystagmus Syndrome and Controls. Optom. Vis. Sci 95(4): 373-383.

8. Simons,D. J (2000) Attentional capture and inattentional blindness. Trends Cogn. Sci 4(4): 147-155.

9. Sharek D (2011) A Useable, Online NASA-TLX Tool. Proc Hum Factors Ergon Soc 55(1): 1375-1379.

10. Bieg H. J, Bresciani J. P, Bülthoff H. H, Chuang L. L (2012) Looking for discriminating is different from looking for looking's sake. PLoS One 7(9): e45445.

11. Corbetta M, Akbudak E, Conturo T. E, Snyder A. Z, Ollinger J. M, Drury H. A, Linenweber M. R, Petersen S. E, Raichle M. E, Van Essen D. C (1998) A common network of functional areas for attention and eye movements. Neuron 21(4): 761-773.

12. Simon O, Mangin J. F, Cohen L, Le Bihan, D, Dehaene S (2002) Topographical layout of hand, eye, calculation, and language-related areas in the human parietal lobe. Neuron 33(3): 475-487.

13. Hoffman J. E, Subramaniam B (1995) The role of visual attention in saccadic eye movements. Percept Psychophys 57(6): 787-795.

14. Kowler E, Anderson E, Dosher B, Blaser E (1995) The role of attention in the programming of saccades. Vis. Res 35(13): 1897-1916. https://doi.org/10.1016/0042-6989(94)00279-u

15. Knops A, Thirion B, Hubbard E. M, Michel V, Dehaene S (2009) Recruitment of an area involved in eye movements during mental arithmetic. Science 324(5934): 1583-1585.

https://doi.org/10.1126/science.1171599

16. Duncan J, Humphreys G, Ward R (1997) Competitive brain activity in visual attention. Curr. Opin. Neuro/7(2): 255-261.

17. Halliday J, Carpenter R. H (2010) The effect of cognitive distraction on saccadic latency. Perception 39(1): 41-50.

18. Montagnini A, Chelazzi L (2005) The urgency to look: prompt saccades to the benefit of perception. Vis. Res 45(27): 3391-3401.

19. Pashler H, Carrier M, Hoffman J (1993) Saccadic eye movements and dual-task interference. Q J Exp Psychol 46(1): 51-82.

20. Van Stockum S, MacAskill M. R, Myall D, Anderson T. J (2011) A perceptual discrimination task abnormally facilitates reflexive saccades in Parkinson's disease. Eur. J. Neurosci 33(11): 2091-2100.

21. Kapoula Z, Robinson D (1986) Saccadic undershoot is not inevitable: Saccades can be accurate. Vis. Res 26(5): 735-743.

22. Weber R. B, Daroff R. B (1972) Corrective movements following refixation saccades: Type and control system analysis. Vis. Res 12(3): 467-475. 
23. Zhai X, Enderle J. D (2016) Visually Guided Horizontal Saccades under the Double-Step Paradigm. Journal of Biomedical Engineering and Medical Devices 10: 1-7.

24. Toyomura A, Omori T (2004) Saccadic undershoot can be explained as a trade-off between accuracy and flight-time. Proceedings of the ISCIE International Symposium on Stochastic Systems Theory and its Applications 130-134.

25. Wang Z, Dell'Osso L (2007) Being "slow to see" is a dynamic visual function consequence of infantile nystagmus syndrome: model predictions and patient data identify stimulus timing as its cause. Vis. Res 47(11): 1550-1560.

26. Dunn M. J, Margrain T. H, Woodhouse J. M, Erichsen J. T (2015) Visual processing in infantile nystagmus is not slow. Invest Ophthalmol Vis Sci 56(9): 5094-5101.

27. Flehmig H. C, Steinborn M. B, Westhoff K, Langner R (2010) Neuroticism and speed-accuracy tradeoff in self-paced speeded mental addition and comparison. J Individ Differ.

28. Salvia E, Guillot A, Collet C (2013) The effects of mental arithmetic strain on behavioral and physiological responses. J Psychophysiol.

29. Reddi B, Asrress K. N, Carpenter R. H (2003) Accuracy, information, and response time in a saccadic decision task. J. Neurophysio/ 90(5): 3538-3546.

30. Reddi B, Carpenter R. H (2000) The influence of urgency on decision time. Nat. Neurosci 3(8): 827.

31. Guyader N, Malsert J, Marendaz C (2010) Having to identify a target reduces latencies in prosaccades but not in antisaccades. Psychological Research PRPF 74(1): 12-20.

32. Kristjánsson Á, Chen Y, Nakayama K (2001) Less attention is more in the preparation of antisaccades, but not prosaccades. Nat. Neurosci 4(10): 1037.

33. Recarte M. A, Nunes L. M (2003) Mental workload while driving: effects on visual search, discrimination, and decision making. J. Exp. Psychol: Applied 9(2): 119.

34. Tsai Y.F, Viirre E, Strychacz C, Chase B, Jung T. P (2007) Task performance and eye activity: predicting behavior relating to cognitive workload. Aviat Space Environ Med 78(5): B176-B185.

35. Henderson J. M, Graham P. L (2008) Eye movements during scene viewing: evidence for mixed control of fixation durations. Psychon Bull Rev 15(3): 566-573. https://doi.org/10.3758/pbr.15.3.566

36. Luke S. G, Nuthmann A, Henderson J. M (2013) Eye movement control in scene viewing and reading: evidence from the stimulus onset delay paradigm. J Exp Psychol Hum Percept Perform 39(1): 10-15. https://doi.org/10.1037/a0030392

37. Nuthmann A (2017) Fixation durations in scene viewing: Modeling the effects of local image features, oculomotor parameters, and task. Psychon Bull Rev 24(2): 370-392. https://doi.org/10.3758/s13423-016-1124-4

38. Kustov A. A, Robinson D. L (1996) Shared neural control of attentional shifts and eye movements. Nature 384(6604): 74.

\section{Figures}




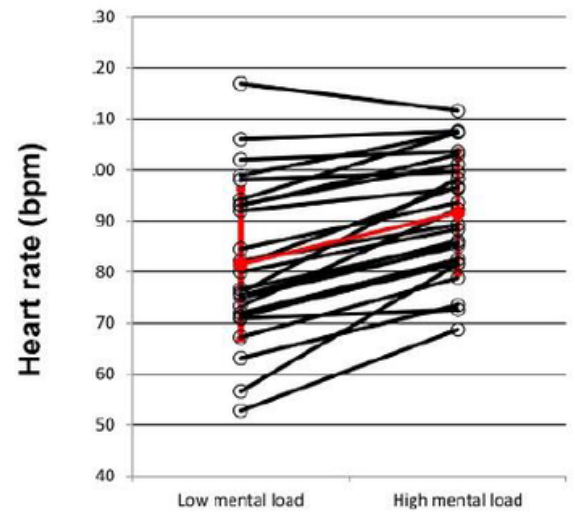

(a)

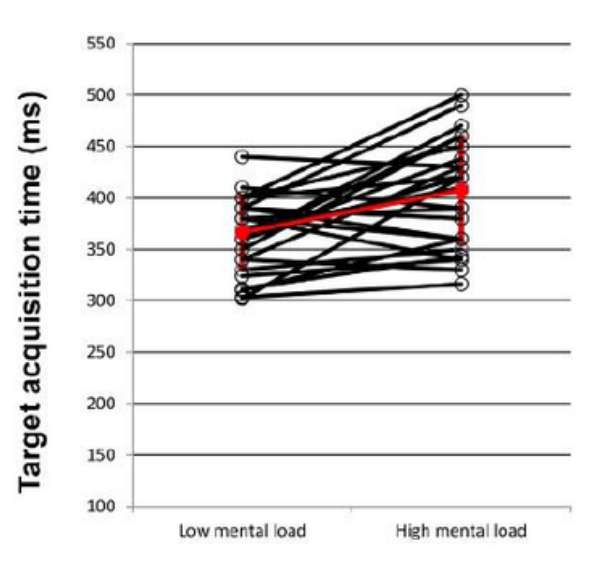

(d)

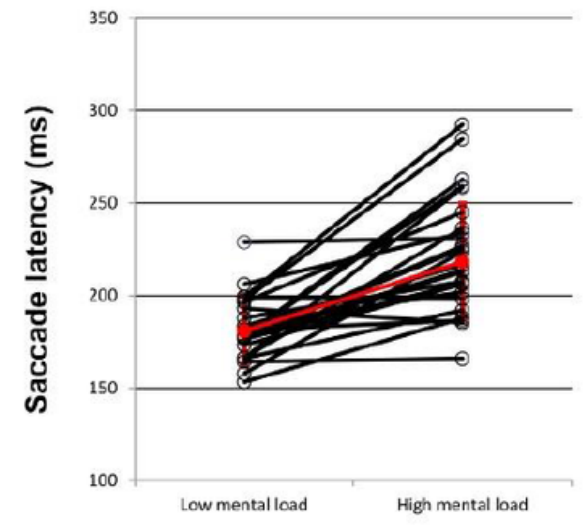

(b)

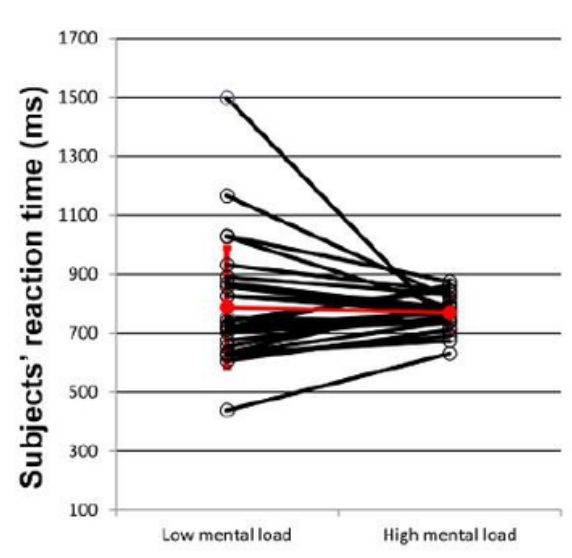

(e)

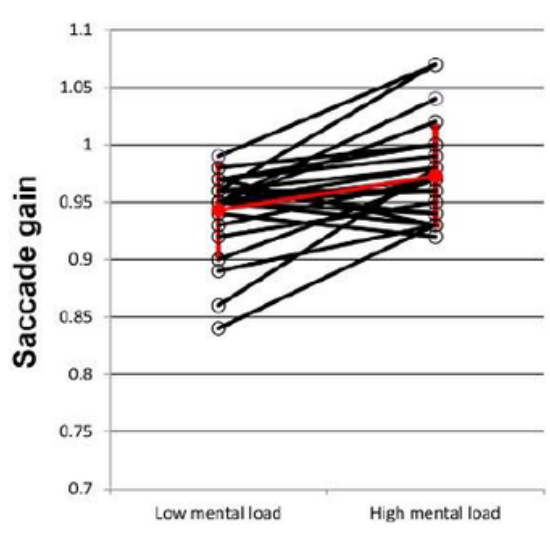

(c)

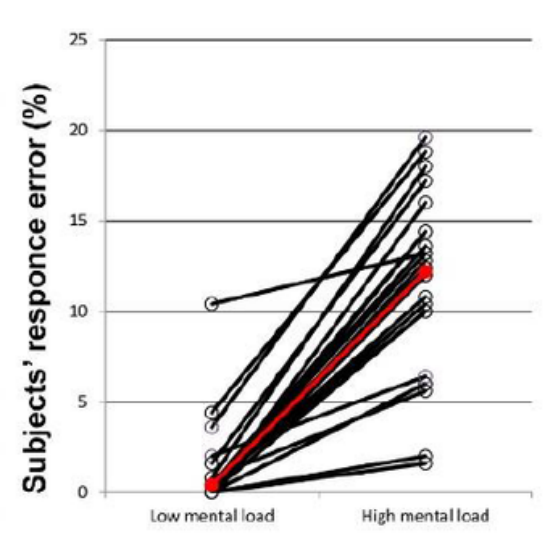

(f)

\section{Figure 1}

Heart rate (a), saccade latency (b), saccade gain (c), latency of target acquisition; i.e., fixation on target of interest (d), participants' reaction time (e) and response errors (f) in post saccadic discrimination task under low and high cognitive loads. Black lines represent changes from low to high cognitive load for each participant. Red circles with vertical lines express the mean \pm SD under the low and high loads; except for response errors that the red circles express median values. Red diagonal lines represent changes in the means for figures (a-e) and changes in the medians for figure (f) from low to high cognitive load. 


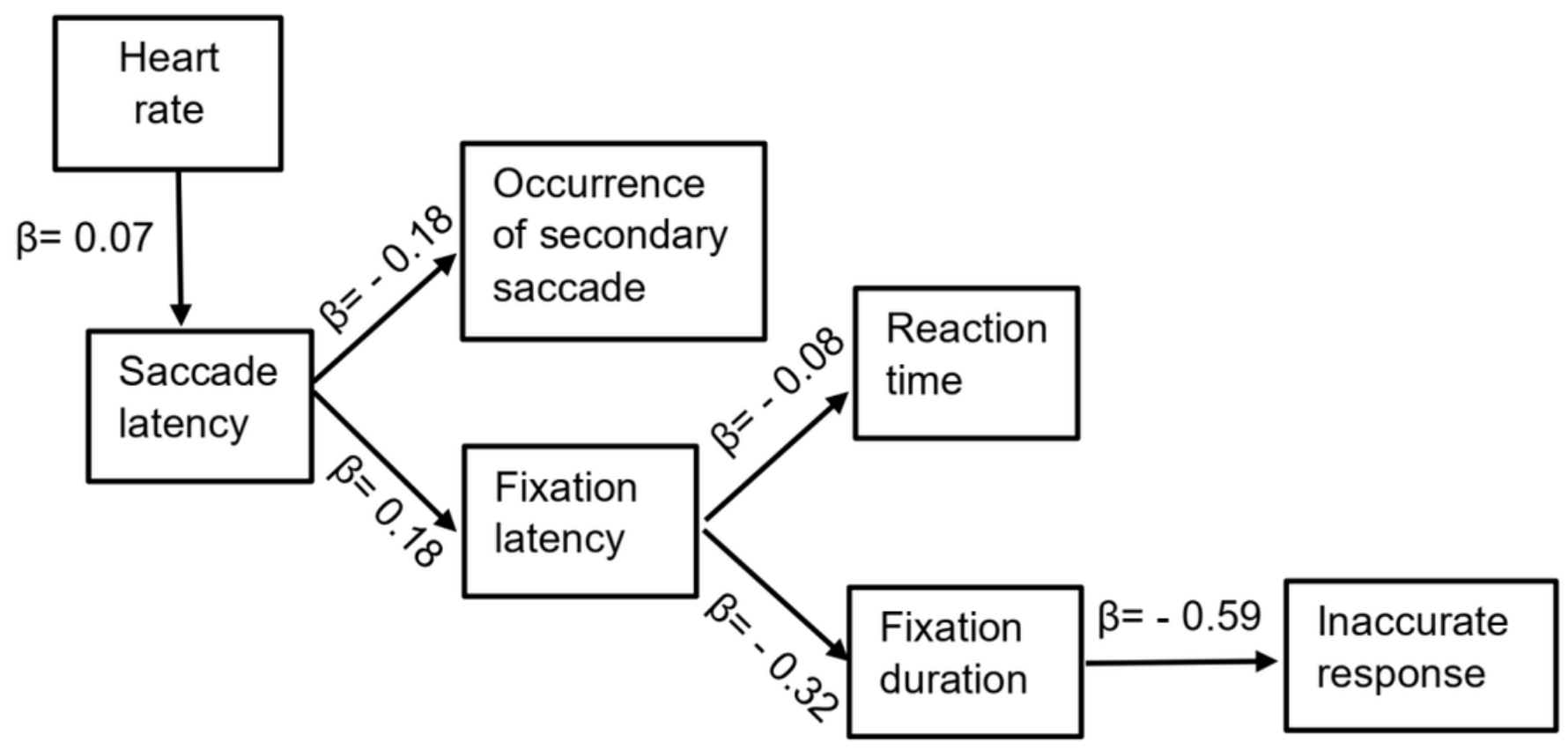

Figure 2

Illustration of final Path Models for the indirect effects of the changes in the cognitive load indicated by heart rate, from low to high load on subjects' reaction time and submission of inaccurate responses. 